\title{
LHCb: Status and Prospects on the $b$ Anomalies
}

\author{
A. Hicheur*i \\ Federal University of Rio de Janeiro \\ On behalf of LHCb Collaboration \\ E-mail: Adlene.Hicheurecern.ch
}

Since the start of the Large Hadron Collider program, direct searches for Beyond Standard Model (BSM) particles have constrained their mass scale to limits which are now above the energy reach of the current collider. As a result, studies of indirect probes of BSM physics have gained a considerable momentum, both experimentally and theoretically. The flavour anomalies in $b$ hadron decays are now recognized as an important laboratory for the indirect detection of BSM physics. This short review presents several key analyses in this area, and some prospects with future data.

BEAUTY2020

21-24 September 2020

Kashiwa, Japan (online)

${ }^{*}$ Speaker.

${ }^{\dagger}$ also at U. Constantine, Algeria. 


\section{Introduction}

Heavy Flavour decays are usually described by low energy effective Hamiltonians forming an Effective Field Theory (EFT) (see, e.g. reference [1] for a review). The Hamiltonians are written as:

$$
H=\sum_{i} V_{C K M}^{i} C_{i}(\mu) O_{i}(\mu)
$$

where $C_{i}(\mu)$ are the Wilson coefficients integrating out the physics above the scale $\mu$ (short range), $O_{i}(\mu)$ are current operators whose matrix elements represent the low energy (non-perturbative/long range) hadronic physics, and $\mu$ is the renormalization scale (typically $\sim 1 \mathrm{GeV}$ ) distinguishing the two regimes. $V_{C K M}^{i}$ represents the flavour coupling associated to an operator $O_{i}$, i.e., CabibboKobayashi-Maskawa (CKM) matrix elements for SM operators. The Wilson coefficients thus represent the quantities which are impacted by the intervention of BSM physics.

For the semileptonic tree decays, the coupling of the mediating $W$ boson does not discriminate between lepton flavours in the SM. On the contrary, a BSM mediator might exhibit different couplings between light and heavy leptons. This is referred to as Lepton Flavour Universality Violation (LFUV). Such an effect could also occur for the semileptonic loop decays $b \rightarrow s \ell \ell$ where the dominant operators are $O_{7}, O_{9}, O_{10}$. Loop decays could also be the ground of new dynamics involving Lepton Flavour Violation (LFV) where leptons of different flavours are produced together. A review of various LHCb analyses is described below. Several of them exhibit deviations from SM that can be explained consistently with theoretical models.

\section{Semileptonic tree decays}

The dominant decays $\left(b \rightarrow c \ell^{-} \bar{v}\right)$ of this kind are generically written as $H_{b} \rightarrow H_{c} \ell^{-} \bar{v}$ where $H_{b}$ is a $b$ hadron and $H_{c}$ is a charm hadron. The search for a possible LFUV is performed through the measurement of the ratio:

$$
R\left(H_{c}\right)=\frac{\mathscr{B}\left(H_{b} \rightarrow H_{c} \tau^{-} \bar{v}\right)}{\mathscr{B}\left(H_{b} \rightarrow H_{c} \mu^{-} \bar{v}\right)},
$$

where $\mathscr{B}$ denotes the branching fraction. A BSM mediating heavy boson might couple preferentially to the tau lepton, as in Fig.1, and thus produce a $R\left(H_{c}\right)$ ratio different from the expected SM-based calculations.

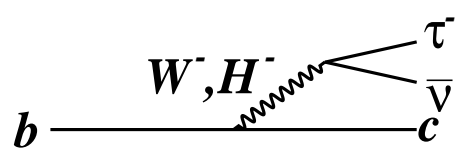

Figure 1: $b \rightarrow c \tau^{-} \bar{v}$ semileptonic transition with a mediating $W$ or charged Higgs boson as derived from 2HDM models discussed e.g. in Ref. [2].

The modes $\bar{B}^{0} \rightarrow D^{+(*)} \ell^{-} \bar{v}$ have drawn attention both at the $b$ factories and the LHCb experiment. The LHCb collaboration has been focusing so far on $R\left(D^{*+}\right)$, where $D^{*+}$ is reconstructed via $D^{*+} \rightarrow D^{0}\left(\rightarrow K^{-} \pi^{+}\right) \pi^{+}$. The $\tau$ lepton is reconstructed in the muonic mode, $\tau^{-} \rightarrow \mu^{-} \bar{v}_{\mu} v_{\tau}$ [3], or the hadronic mode $\tau \rightarrow \pi \pi \pi\left(\pi^{0}\right) v_{\tau}$ [4]. The discriminating variables include the missing mass, 
$m_{\text {miss }}^{2}=\left(P_{B}-P_{D^{*}}-P_{\mu}\right)^{2}$, the momentum transfer $q^{2}=\left(P_{B}-P_{D^{*}}\right)^{2}$, the muon energy $E_{\mu}^{*}$, the $\tau$ lifetime (for the hadronic mode) and a Boost Decision Trees classifier [5] to reject double-charm decays of the type $B \rightarrow D D X$ (for the hadronic mode). The muonic tau analysis [3] obtained a measurement of $R\left(D^{*+}\right)=0.336 \pm 0.027$ (stat) \pm 0.030 (syst) while the hadronic tau study [4] gives $R\left(D^{*+}\right)=0.291 \pm 0.021$ (stat) \pm 0.026 (syst) $\pm 0.013(B F)$, where the last uncertainty is due to the branching fraction of the normalizing channel $\bar{B}^{0} \rightarrow D^{*+} \pi^{-} \pi^{+} \pi^{-}$. The latest HFLAV averaging [6] in the $R(D)-R\left(D^{*}\right)$ plane, including the recent Belle collaboration $R\left(D^{(*)}\right)$ measurements [7], is shown in Fig.2. Compared to an averaged series of SM-based predictions [8], a discrepancy of 3.1 $\sigma$ is observed.

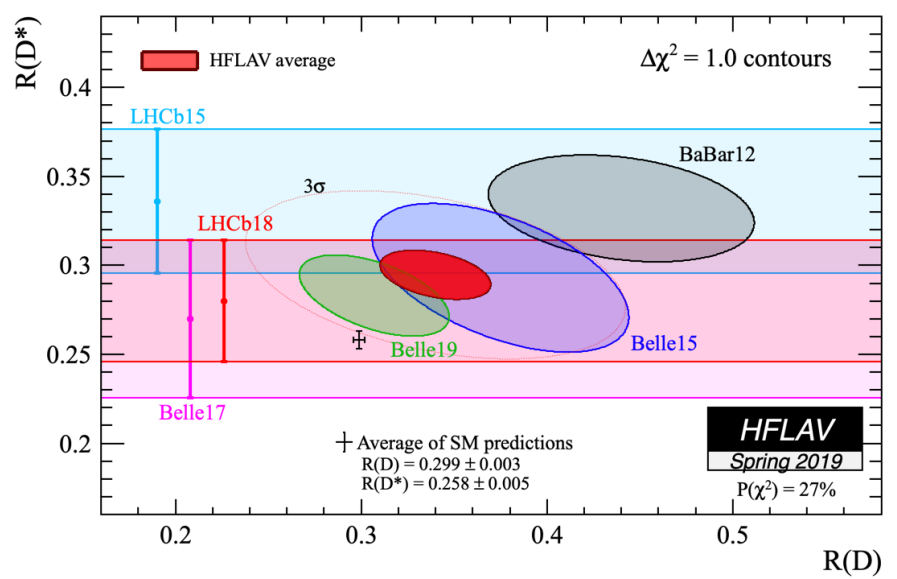

Figure 2: $R(D)$ and $R\left(D^{*}\right)$ measurements compared to the SM average [6].

A similar measurement with the decays $B_{c}^{-} \rightarrow J / \psi \ell^{-} \bar{v}, R(J / \psi)$, has been performed recently by the $\mathrm{LHCb}$ collaboration for the $\tau$ muonic mode [9], leading to $R(J / \psi)=0.71 \pm 0.17$ (stat) \pm 0.18 (syst) which lies $2 \sigma$ above the range of the known theoretical estimates [10].

\section{3. $b \rightarrow$ sll transitions}

At quark level, $b \rightarrow$ sl $\ell$ transitions proceed through the diagrams shown in Fig.3. The operators contributing to these decays are not evenly distributed in the $q^{2}=m_{\ell \ell}^{2}$ range: at low $q^{2}, O_{7}$ dominates (for transitions to non-scalar hadrons), in the central $q^{2}$ region below the charmonium resonances, $O_{7}$ and $O_{9}$ interfere, and at high $q^{2} O_{9}$ and $O_{10}$ interfere. At the hadron level, the modes investigated by $\mathrm{LHCb}$ are $B^{+} \rightarrow K^{*+} \ell^{+} \ell^{-}, B^{0} \rightarrow K^{0} \ell^{+} \ell^{-}, B^{0} \rightarrow K^{* 0} \ell^{+} \ell^{-}, B_{s}^{0} \rightarrow \phi \ell^{+} \ell^{-}$, and $\Lambda_{b}^{0} \rightarrow \Lambda \ell^{+} \ell^{-}$ (charge conjugation is implied).

A series of studies [11] have dealt with the dynamics of the muonic modes, $\ell=\mu$, to infer the differential decay rate $\frac{d \Gamma}{d q^{2}}$, as illustrated in Fig.4. The data are systematically below the SM-based theoretical predictions, with local discrepancies exceeding $3 \sigma$. Attempting to explore this intriguing behaviour, angular analyses were performed for $B^{0} \rightarrow K^{* 0} \mu^{+} \mu^{-}[11](\mathrm{c}), B_{s}^{0} \rightarrow \phi \mu^{+} \mu^{-}[11]$ (b) and $\Lambda_{b}^{0} \rightarrow \Lambda \mu^{+} \mu^{-}$[12]. Quantities such as $P_{5}^{\prime}=\frac{S_{5}}{\sqrt{F_{L}\left(1-F_{L}\right)}}$ have been built to reduce the hadronic uncertainties [13] from the coefficients $S_{5}$ and $F_{L}$ (fraction of the $K^{*}$ longitudinal polarization) of the angular distribution. For $B^{0} \rightarrow K^{* 0} \mu^{+} \mu^{-}$, the discrepancy reported in previous studies for $P_{5}^{\prime}$ 

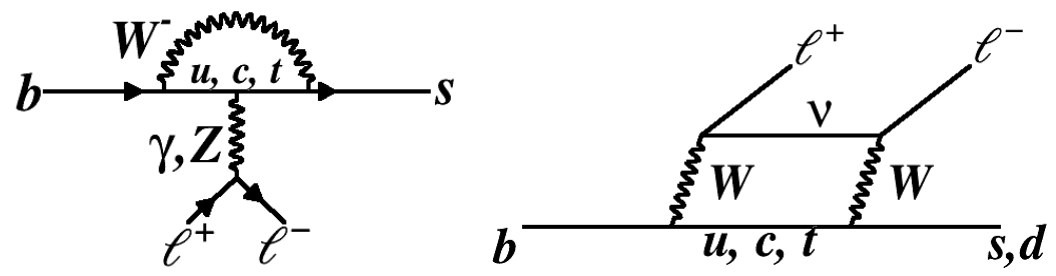

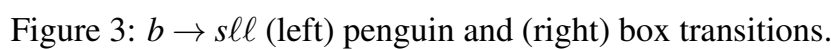
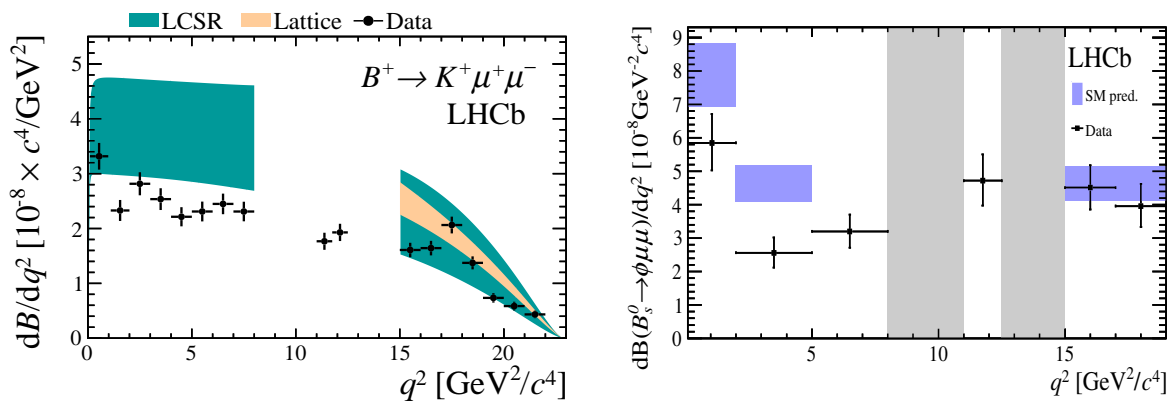

Figure 4: $\frac{d \Gamma}{d q^{2}}$ distribution for (left) $B^{+} \rightarrow K^{+} \mu^{+} \mu^{-}$and (right) $B_{s}^{0} \rightarrow \phi \mu^{+} \mu^{-}$. The $J / \psi$ and $\psi(2 S) q^{2}$ regions are excluded. The points represent the data measurements and the rectangles or bands represent the SM-based predictions.
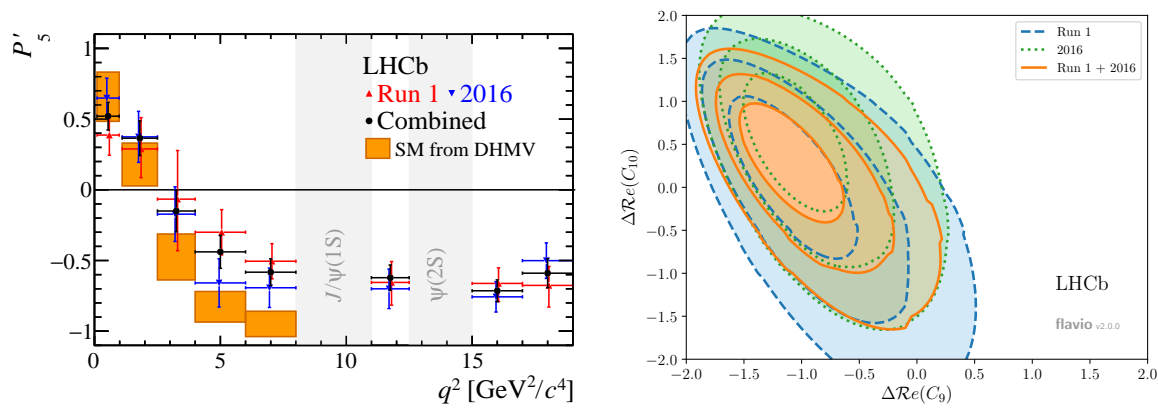

Figure 5: (left) Evolution of $P_{5}^{\prime}$ (see text) as a function of $q^{2}$ for $B^{0} \rightarrow K^{* 0} \mu^{+} \mu^{-}$and (right) resulting 1,2,3 $\sigma$ contours, using all the angular variables, of the deviations from SM of the real parts of the $C_{9}$ and $C_{10}$ Wilson coefficients.

seems to be persistent as shown in Fig.5(left). The fit for the deviations from SM to the real parts of the $C_{9}$ and $C_{10}$ Wilson coefficients gives the results depicted in Fig.5(right). Considering only $C_{9}$, the deviation from SM is determined to be $3.3 \sigma$.

Another way to probe the presence of New Physics is to measure the ratio $R_{X}=\frac{\mathscr{B}\left(H_{b} \rightarrow X \mu^{+} \mu^{-}\right)}{\mathscr{B}\left(H_{b} \rightarrow X e^{+} e^{-}\right)}$, where $X$ denotes a hadronic system comprising a strange quark. The LHCb collaboration studied $R_{K}\left(B^{+} \rightarrow K^{+} \ell^{+} \ell^{-}\right)$[14], $R_{K^{*}}\left(B^{0} \rightarrow K^{* 0} \ell^{+} \ell^{-}\right)$[15] and $R_{p K}$ with the decay $\Lambda_{b}^{0} \rightarrow p K^{-} \ell^{+} \ell^{-}$[16]. For $R_{K}$, the explored $q^{2}$ range is $[1.1,6] \mathrm{GeV}^{2} / c^{4}$, i.e. below the charmonium radiative tails, and above backgrounds of the type $B^{+} \rightarrow K^{+} \phi\left(\rightarrow \ell^{+} \ell^{-}\right)$. The $R_{K^{*}}$ analysis uses two bins in $q^{2}$, $[0.045,1.1] \mathrm{GeV}^{2} / c^{4}$ (above the photon pole) and $[1.1,6] \mathrm{GeV}^{2} / c^{4}$. Finally, $R_{p K}$ is measured with the requirements $q^{2} \in[0.1,6.0] \mathrm{GeV}^{2} / c^{4}$ and $m(p K)<2.6 \mathrm{GeV} / c^{2}$.

The obtained measurements are $R_{K}=0.846_{-0.054}^{+0.060}(\text { stat })_{-0.014}^{+0.016}($ syst $)\left(1.1<q^{2}<6 \mathrm{GeV}^{2} / c^{4}\right)$; 
$R_{K^{*}}=0.66_{-0.07}^{+0.11}($ stat $) \pm 0.03$ (syst) for $0.045<q^{2}<1.1 \mathrm{GeV}^{2} / c^{4}$ and $0.69_{-0.07}^{+0.11}($ stat $) \pm 0.05$ (syst) for $1.1<q^{2}<6 \mathrm{GeV}^{2} / c^{4}$. All these values are systematically below the SM-based predictions by $2.2 \sigma$ to $2.5 \sigma$ [17]. With the $\Lambda_{b}^{0} \rightarrow p K^{-} \ell^{+} \ell^{-}$decay, a first observation of $\Lambda_{b}^{0} \rightarrow p K^{-} e^{+} e^{-}$is obtained with a similar Run 1 and part of Run 2 data set, as illustrated in Fig.6, leading to the measurement of the ratio $R_{p K}=0.86_{-0.11}^{+0.14}($ stat $) \pm 0.05$ (syst). For all these measured quantities, the uncertainty will be reduced soon with the addition of the remainder of Run 2 data.
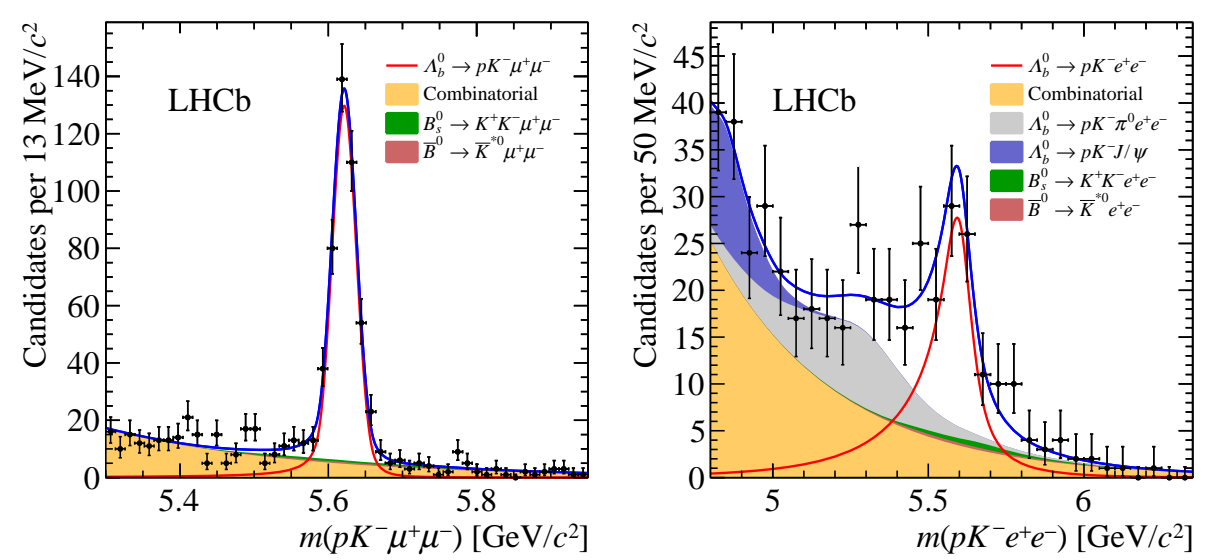

Figure 6: Invariant mass distributions of (left) $\Lambda_{b}^{0} \rightarrow p K^{-} \mu^{+} \mu^{-}$and (right) $\Lambda_{b}^{0} \rightarrow p K^{-} e^{+} e^{-}$candidates. The fit shapes of the $\Lambda_{b}^{0} \rightarrow p K^{-} \ell^{+} \ell^{-}$signals and the main backgrounds are overlaid.

Figure 7 shows the impact of the $R_{K^{*}}$ measurements by BaBar and LHCb, as well as the combination of the LFUV and angular parameters from all the experiments, on the New Physics contributions to the Wilson coefficients $C_{9}$ and $C_{10}$, as derived in Ref. [18]. Combining all anomalous data, $C_{9}$ departs by more than $6 \sigma$ from its SM-based prediction.
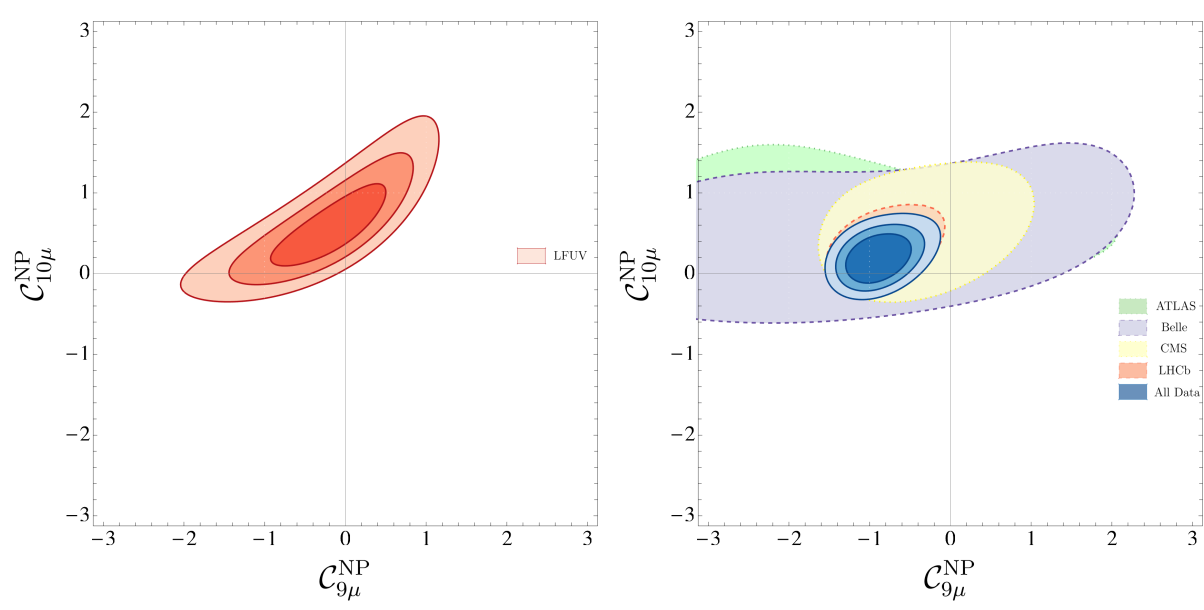

Figure 7: The 1,2,3 $\sigma$ contours for the New Physics contributions to the $C_{9}$ and $C_{10}$ Wilson coefficients using (left) only the LFUV data from Belle and LHCb and (right) combining all data from angular analyses and LFUV. The fits are provided in Ref. [18]. 
Table 1: Expected precisions for $R_{X}=\mathscr{B}\left(H_{b} \rightarrow X \mu^{+} \mu^{-}\right) / \mathscr{B}\left(H_{b} \rightarrow X e^{+} e^{-}\right)$. The numbers are taken from Ref [28] and are projections based on Run 1 results where statistical and systematic uncertainties are combined.

\begin{tabular}{l|cccc}
\hline$R_{X}$ & Run $1 \& 2\left(9 \mathrm{fb}^{-1}\right)$ & Run 3 $\left(23 \mathrm{fb}^{-1}\right)$ & Run 4 $\left(50 \mathrm{fb}^{-1}\right)$ & Run 5 $\left(300 \mathrm{fb}^{-1}\right)$ \\
\hline$R_{K}$ & 0.043 & 0.025 & 0.017 & 0.007 \\
$R_{K^{* 0}}$ & 0.052 & 0.031 & 0.020 & 0.008 \\
$R_{\phi}$ & 0.130 & 0.076 & 0.050 & 0.020 \\
$R_{p K}$ & 0.105 & 0.061 & 0.041 & 0.016 \\
$R_{\pi}$ & 0.302 & 0.176 & 0.117 & 0.047 \\
\hline \hline
\end{tabular}

\section{4. $B \rightarrow \ell \ell$}

For the purely leptonic modes, the combination of the most recent results of the ATLAS [19], CMS [20] and LHCb [21] experiments, giving $\mathscr{B}\left(B_{s}^{0} \rightarrow \mu^{+} \mu^{-}\right)=\left(2.69_{-0.35}^{+0.37}\right) \times 10^{-9}$ and $\mathscr{B}\left(B^{0} \rightarrow \mu^{+} \mu^{-}\right)<1.9 \times 10^{-10}$ at $95 \%$ confidence level $(\mathrm{CL})$, shows that $B_{s}^{0} \rightarrow \mu^{+} \mu^{-}$is $2 \sigma$ below the SM-based predictions. First attempts by LHCb of measuring the ditauon [22] and dielectron [23] modes led to the results $\mathscr{B}\left(B_{s}^{0} \rightarrow \tau^{+} \tau^{-}\right)<6.3 \times 10^{-3}, \mathscr{B}\left(B^{0} \rightarrow \tau^{+} \tau^{-}\right)<2.1 \times 10^{-3}$, $\mathscr{B}\left(B_{s}^{0} \rightarrow e^{+} e^{-}\right)<11.2 \times 10^{-9}$ and $\mathscr{B}\left(B^{0} \rightarrow e^{+} e^{-}\right)<3.0 \times 10^{-9}$ at $95 \% \mathrm{CL}$.

\section{LFV searches}

The hints of LFUV in $b \rightarrow$ sll decays have motivated recent LFV searches, seeking to observe decays of the type $b \rightarrow s \ell \ell^{\prime}$ or $B \rightarrow \ell \ell^{\prime}$. A first study of $B^{+} \rightarrow K^{+} \mu^{ \pm} e^{\mp}$ [24] led to the establishment of the 95\% CL limits: $\mathscr{B}\left(B^{+} \rightarrow K^{+} \mu^{-} e^{+}\right)<9.5 \times 10^{-9}$ and $\mathscr{B}\left(B^{+} \rightarrow K^{+} \mu^{+} e^{-}\right)<8.8 \times 10^{-9}$. Another analysis on $B^{+} \rightarrow K^{+} \mu^{-} \tau^{+}$[25], characterized by the original use of the decay $B_{s 2}^{* 0} \rightarrow B^{+} K^{+}$ to constraint the $\tau$ four-momentum, obtained the less stringent limit $\mathscr{B}\left(B^{+} \rightarrow K^{+} \mu^{-} \tau^{+}\right)<4.5 \times$ $10^{-5}$. For what concerns the LFV leptonic modes, the decays $B_{(s)}^{0} \rightarrow e^{ \pm} \mu^{\mp}[26]$ and $B_{(s)}^{0} \rightarrow \tau^{ \pm} \mu^{\mp}$ [27] have been studied, setting the $95 \%$ CL limits to $\mathscr{B}\left(B_{s}^{0} \rightarrow e^{ \pm} \mu^{\mp}\right)<6.3 \times 10^{-9}, \mathscr{B}\left(B^{0} \rightarrow\right.$ $\left.e^{ \pm} \mu^{\mp}\right)<1.3 \times 10^{-9}, \mathscr{B}\left(B_{s}^{0} \rightarrow \tau^{ \pm} \mu^{\mp}\right)<4.2 \times 10^{-5}$ and $\mathscr{B}\left(B^{0} \rightarrow \tau^{ \pm} \mu^{\mp}\right)<1.4 \times 10^{-5}$.

\section{Prospects and summary}

Most analyses presented have been published on a partial LHCb data set and are currently being updated. The second column of Table 1 shows the expected precisions for the $R_{X}$ measurements for the full Run1+Run2 statistics.

On the longer term, the third, fourth and fifth columns of Table 1 show the evolution of the expected sensitivities for the future runs of data taking. For the tree semileptonic decays, the LFU ratios $R\left(D^{0}\right), R\left(D^{+}\right), R\left(D_{s}^{(*)}\right), R\left(\Lambda_{c}^{(*)}\right), R(J / \psi)$ and $R(p)$ (from $\Lambda_{b}^{0} \rightarrow p \tau \nu$ ) are foreseen during the first phase of Run 3. Figure 8 shows the evolution of the $R\left(H_{c}\right)$ ratios throughout the periods of data taking.

The available results on the anomalies in the $b$-hadron decays show a combination of deviations, which has triggered an intense activity on the phenomenological side in studies aiming at constraining the Wilson coefficients and probing possible contributions of New Physics [18, 30,31]. The 6 to 


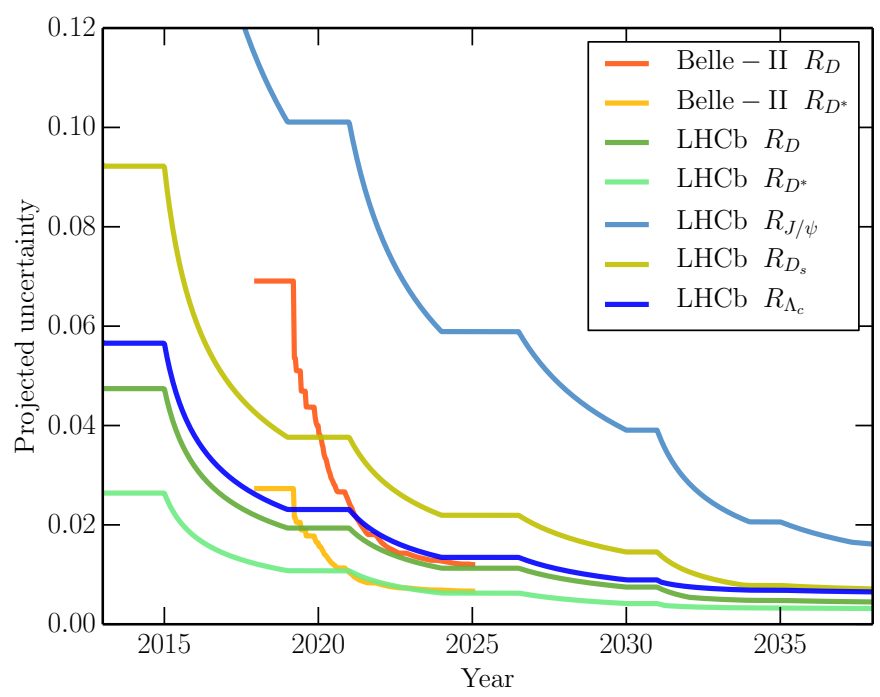

Figure 8: Evolution of the sensitivity on the semileptonic ratios $R\left(H_{c}\right)$ as reported in Ref. [29].

$7 \sigma$ deviation from SM derived for $C_{9}$ is subject to interpretations, which try to account for what is observed in both $b \rightarrow c$ tree transitions and $b \rightarrow s$ loop decays. Explanations based on vector Leptoquarks [32] and the "4321" model [33] have become popular. Any explored paradigm will have to satisfy the constraints of $B_{s}^{0}$ meson mixing and the $B_{c}^{+}$meson lifetime.

\section{References}

[1] G. Buchalla et al., Rev. Mod. Phys. 68 (1996) 1125-1144

[2] J.F. Gunion, E.H. Haber, G.L. Kane, S. Dawson, The Higgs Hunter's Guide, Front.Phys.80 (2000) 1-404

[3] LHCb collaboration, R. Aaij et al., Phys. Rev. Lett. 115 (2015) 111803

[4] LHCb collaboration, R. Aaij et al., Phys. Rev. Lett. 120 (2018) 171802

[5] L. Breiman, J.H. Friedman, R.A. Olshen and C.J. Stone, Classification and regression trees, Wadsworth international group (1984) Belmont, California, USA

[6] Heavy Flavor Averaging Group, Y. Amhis et al., arXiv:1909.12524, latest results and plots available at https://hflav.web.cern.ch/

[7] Belle Collaboration, A. Abdesselam et al., arXiv:1904.08794

[8] D. Bigi, P. Gambino, Phys. Rev. D94 (2016) 094008; F.Bernlochner et al., Phys. Rev. D95 (2017) 115008 ; D.Bigi et al., JHEP 1711 (2017) 061; S.Jaiswal et al., JHEP 1712 (2017) 060

[9] LHCb collaboration, R. Aaij et al., Phys. Rev. Lett. 120 (2018) 121801

[10] A.Yu.Anisimov et al., Phys. Lett. B452 (1999) 129; M.A.Ivanov et al., Phys. Rev. D73 (2006) 054024; E.Hernandez et al., Phys. Rev. D74 (2006) 074008

[11] LHCb collaboration, R. Aaij et al., a: JHEP 06 (2014) 133; b: JHEP 09 (2015) 179; c: PRL 125 (2020) 011802; d: JHEP 06 (2015) 115

[12] LHCb collaboration, R. Aaij et al., JHEP 09 (2018) 146 
[13] Descotes-Genon et al., JHEP 05 (2013) 137

[14] LHCb collaboration, R. Aaij et al., Phys. Rev. Lett. 122 (2019) 191801

[15] LHCb collaboration, R. Aaij et al., JHEP 08 (2017) 055

[16] LHCb collaboration, R. Aaij et al., JHEP 05 (2020) 040

[17] Non-exhaustive: C. Bobeth et al. JHEP 07 (2007) 040; M.Bordone et al., Eur. Phys. J. C76 (2016) 440; W. Altmannshofer et al., Phys. Rev. D96 (2017) 055008

[18] M. Alguero et al., Eur. Phys. J. C79 (2019) 8, 714; Eur. Phys. J.C80 (2020) 6, 511 (addendum).

[19] ATLAS collaboration, ATLAS-CONF-2020-049

[20] CMS collaboration, CMS-PAS-BPH-20-003

[21] LHCb collaboration, LHCb-CONF-2020-002

[22] LHCb collaboration, R. Aaij et al., Phys. Rev. Lett. 118 (2017) 251802

[23] LHCb collaboration, R. Aaij et al., Phys. Rev. Lett. 124 (2020) 211802

[24] LHCb collaboration, R. Aaij et al., Phys. Rev. Lett. 123 (2019) 241802

[25] LHCb collaboration, R. Aaij et al., JHEP 06 (2020) 129

[26] LHCb collaboration, R. Aaij et al., JHEP 03 (2018) 078

[27] LHCb collaboration, R. Aaij et al., Phys. Rev. Lett. 123 (2019) 211801

[28] LHCb collaboration, R. Aaij et al., arXiv:1808.08865, LHCB-PUB-2018-009, CERN-LHCC-2018-027

[29] S. Bifani et al., J. Phys. G: Nucl. Part. Phys. 46 (2019) 023001

[30] J. Aebischer et al., arXiv:1903.10434

[31] D. M. Straub, flavio package, arXiv:1810.08132

[32] D. Buttazzo et al., JHEP 11 (2017) 044

[33] L. Di Luzio et al., Phys. Rev. D96, (2017) 115011; JHEP 11 (2018) 081 